\title{
Gonorrhée disséminée associée à une atteinte du larynx chez un homme de 25 ans
}

\author{
François Voruz MD, Igor Leuchter MD
}

Citation : CMAJ 2021 May 3;193:E646. doi : 10.1503/cmaj.202183-f

Voir la version anglaise de l'article ici : www.cmaj.ca/lookup/doi/10.1503/cmaj.202183
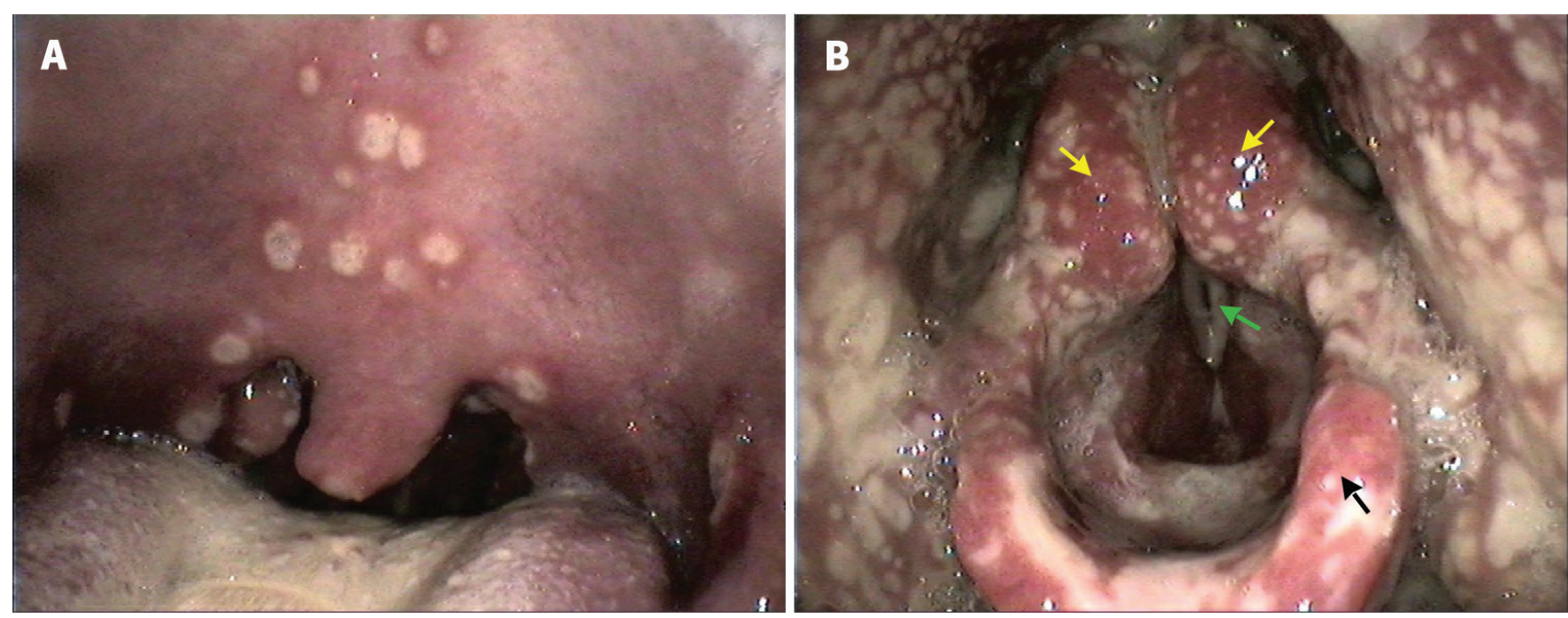

Figure 1 : A) Pharynx d'un homme de 25 ans présentant des macules blanchâtres dispersées et un érythème périlésionnel sur le palais, l'uvule et les amygdales. B) Larynx du patient; on y voit des lésions fibrineuses dispersées impliquant les structures environnantes, sans œdème des cordes vocales. Remarque : Flèches jaunes = aryténoïdes, flèche verte = corde vocale inférieure gauche, flèche noire = épiglotte.

$\mathbf{U}$ n homme marié de 25 ans, en santé et immunocompétent, s'est présenté au service des urgences pour une odynophagie grave présente depuis 5 jours, de la fièvre (pic à $40^{\circ} \mathrm{C}$ ) et une éruption cutanée pustuleuse dispersée. II a déclaré ne pas avoir eu de relations sexuelles extraconjugales. L'examen physique du patient a fait état de lésions fibrineuses dispersées dans le pharynx et le larynx (figure 1; annexe 1 [vidéo], accessible en anglais au www.cmaj.ca/lookup/doi/10.1503/ cmaj.202183/tab-related-content) ainsi que de lésions pustuleuses sur le torse, les membres et le scrotum. Le patient ne présentait pas d'urétrite.

Un test d'amplification en chaîne par polymérase d'une pustule cutanée a révélé la présence de Neisseria gonorrhoeae, ce qui concordait avec un diagnostic d'infection gonococcique disséminée associée à une atteinte du larynx. Le dépistage du VIH, de la syphilis, de la chlamydia et de l'infection au virus herpès simplex s'est avéré négatif. Après une consultation avec notre équipe spécialisée en maladies infectieuses, le patient a été hospitalisé et a reçu des liquides par voie intraveineuse, une alimentation par sonde nasogastrique, du fentanyl et une antibiothérapie intraveineuse à la ceftriaxone. Ses lésions se sont résorbées en 9 jours. Son conjoint ou sa conjointe a obtenu un résultat négatif au dépistage de la gonorrhée et n'a donc pas reçu de traitement. Nous avons été incapables de déterminer la source de l'infection, mais nous avons conclu qu'il était plausible que le patient ait été un porteur asymptomatique de la bactérie.

L'infection gonococcique est une maladie transmissible sexuellement contractée lors d'une relation sexuelle vaginale, anale ou buccogénitale; elle affecte principalement les muqueuses et se manifeste surtout comme une infection génitourinaire localisée ${ }^{1}$. En 2017, au Canada, l'incidence de la gonorrhée était de 79,5 cas pour 100000 personnes, soit plus du double de l'incidence mesurée en $2013^{2}$.

L'infection disséminée découle de la propagation hématogène de la bactérie $N$. gonorrhoeae et se manifeste habituellement sous forme de paraténonite, d'arthrite, de lésions cutanées ou, très rarement, de méningite, d'endocardite ou d'ostéomyélite 3 . Souvent asymptomatique, l'atteinte du pharynx survient dans $3 \%$ des cas; les relations sexuelles buccogénitales sont le facteur de risque le plus important $t^{4,5}$. Le diagnostic 
différentiel comprend l'arthrite infectieuse ou réactionnelle, une infection à herpès simplex, le VIH et la syphilis.

En raison de la résistance de la bactérie à la pénicilline et à la quinolone, on recommande l'administration de céphalosporines de troisième génération, comme la ceftriaxone, mais des souches résistantes préoccupantes sont toutefois en émergence $^{1}$. Lorsque vous évaluez des lésions cutanées, envisagez la possibilité d'une infection transmissible sexuellement, faites une vérification approfondie des antécédents sexuels et recherchez la présence de manifestations systémiques atypiques, comme une pharyngolaryngite.

\section{Références}

1. Hill SA, Masters TL, Wachter J. Gonorrhea - an evolving disease of the new millennium. Microb Cell 2016;3:371-89.

2. Report on sexually transmitted infections in Canada, 2017. Gonorrhea. Ottawa: Public Health Agency of Canada; modified 2020 Jan. 27. Accessible ici : www.canada.ca/en/public-health/services/publications/diseases-conditions /report-sexually-transmitted-infections-canada-2017.html\#a5 (consulté le 29 oct. 2020).

3. Bleich AT, Sheffield JS, Wendel GD Jr., et al. Disseminated gonococcal infection in women. Obstet Gynecol 2012;119:597-602.

4. Chan PA, Robinette A, Montgomery M, et al. Extragenital infections caused by Chlamydia trachomatis and Neisseria gonorrhoeae: a review of the literature. Infect Dis Obstet Gynecol 2016;2016:5758387.

5. Balmelli C, Gunthard HF. Gonococcal tonsillar infection - a case report and literature review. Infection 2003;31:362-5.

Intérêts concurrents : Aucun déclaré.

Cet article a été révisé par des pairs.

Les auteurs ont obtenu le consentement du patient.

Affiliations : Service d'otorhinolaryngologie et de chirurgie cervicofaciale, hôpitaux de l'Université de Genève, Suisse.

Propriété intellectuelle du contenu : Il s'agit d'un article en libre accès distribué conformément aux modalités de la licence Creative Commons Attributions (CC BY-NC-ND 4.0), qui permet l'utilisation, la diffusion et la reproduction dans tout médium à la condition que la publication originale soit adéquatement citée, que l'utilisation se fasse à des fins non commerciales (c.-à-d. recherche ou éducation) et qu'aucune modification ni adaptation n'y soit apportée. Voir : https://creativecommons.org/licenses/by-nc-nd/4.0/deed.fr.

Correspondance : François Voruz, francois.voruz@hcuge.ch

Voir la vidéo connexe en ligne, « Disseminated gonorrhea: scattered fibrinous lesions in the pharynx and larynx ", accessible ici : www.cmaj.ca/lookup/doi/10.1503/cmaj $.202183 /$ tab-related-content. 